\title{
EL DNI ELECTRÓNICO: ECOSISTEMA Y USO EN LA E-ADMINISTRACIÓN LOCAL
}

\author{
Pablo R. Prieto
}

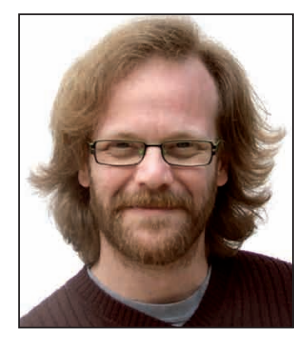

Pablo R. Prieto es doctor en ciencias de la comunicación por la Universidad Rey Juan Carlos (URJC) de Madrid y arquitecto por la Escuela Técnica Superior de Arquitectura de la Universidad Politécnica de Madrid. Es profesor en la Facultad de Ciencias de la Comunicación de la URJC, en el área de conocimiento de periodismo. Es coordinador del Grado en publicidad y relaciones públicas en la modalidad online. Ha participado en proyectos de investigación sobre el diseño de la información, las tecnologías de la información, el diseño de interactivos y la información en la Web. Es miembro del GEAC (Grupo de estudios avanzados de la comunicación) y co-fundador de CODI (Grupo de estudios de la comunicación y diseño de la información), ambos de la URJC.

Fac. de Ciencias de la Comunicación, Univ. Rey Juan Carlos Desp. 227, Edif. Departamental, Campus de Fuenlabrada Camino del Molino, s/n. 28943 Fuenlabrada (Madrid) pablo.prieto@urjc.es

\section{Resumen}

Para que la e-administración local sea una realidad deben existir todos los elementos del ecosistema. Se analiza cuáles son estos elementos, tanto del lado del ciudadano como del lado de la administración, tomando el DNle como centro. Para detectar las carencias y proponer soluciones se cuantifica la realidad actual de cada elemento: desde la existencia de servicios que usen el DNle en los sitios web de los ayuntamientos y las diputaciones provinciales hasta el número de DNle y lectores compatibles en manos de los ciudadanos. El elemento más desfavorable es la poca cantidad de lectores de DNle: según los últimos datos disponibles, sólo un $2 \%$ de la población lo tiene.

\section{Palabras clave}

DNI electrónico, DNle, E-administración local, Ayuntamientos, Diputaciones provinciales, Certificado electrónico, Firma electrónica, Web.

\section{Title: The electronic ID card: its ecosystem and use in local e-Government}

\begin{abstract}
For local e-government to become a reality, all the elements of the ecosystem must be in place. We search for the elements which constitute the ecosystem, both from the citizen and from the city council point of view, with the electronic identity card (eID) as the core. In order to find out the main deficiencies in the system and to propose solutions, we need to quantify the present situation of each element: from city and county councils websites where eID is needed to use the services offered to the number of eID and compatible readers owned by citizens. According to the latest data, only $2 \%$ of the population owns an eID reader: this is the weakest element of our ecosystem.
\end{abstract}

\section{Keywords}

Electronic identity card, eID, E-Government, Local government, City councils, County councils, Digital certificate, Digital signature, Website.

Prieto, Pablo R. "El DNI electrónico: ecosistema y uso en la e-administración local”. El profesional de la información, 2011, mayo-junio, v. 20, n. 3, pp. 276-280.

DOI: 10.3145/epi.2011.may.05

\section{Introducción}

El 16 de marzo de 2006 se puso en marcha el Documento Nacional de Identidad electrónico español (DNle). Y el 22 de junio de 2007 se promulgó la Ley 11/2007 de acceso electrónico de los ciudadanos a los servicios públicos con el objetivo de "reconocer el derecho de los ciudadanos -y no sólo la posibilidad- de acceder mediante comunicaciones electrónicas a la Administración" (BOE, 2007).

La citada Ley establecía el 31 de diciembre de 2009 como la fecha en que la administración local debía hacer efectivo el 
derecho de los ciudadanos a relacionarse con ella utilizando medios electrónicos "en relación con la totalidad de los procedimientos y actuaciones de su competencia". Pero hecha la ley, hecha la trampa: la disposición final tercera también establece que este derecho se hará efectivo "siempre que lo permitan sus disponibilidades presupuestarias".

Dada la obligatoriedad en España de disponer de DNI a partir de los 14 años (Ley orgánica 1/1992, de 21 de febrero), la implantación del DNle y la incorporación en el mismo de dos certificados electrónicos (acreditación de identidad y firma electrónica), la situación auguraba que se efectuaran más trámites telemáticos a través de las administraciones públicas, haciendo realidad la e-administración (Baiget, 2007).

Como demuestran los datos de la investigación que -en parte- aquí se presenta, actualmente al menos el $74 \%$ de la población española tiene acceso a como mínimo un servicio, información o trámite desde la web de su ayuntamiento ante el que puede autentificarse o firmar con su DNIe. Pero esta cifra, en principio buena, necesita de muchos matices.

Conviene repasar cuáles son los elementos que constituyen el ecosistema de la e-administración local para detectar las posibles carencias -que las hay-y proponer soluciones.

Los certificados electrónicos más extendidos en posesión de los ciudadanos son los que incorpora el DNle

\section{Metodología}

El objetivo del estudio fue conocer si los sitios web de los ayuntamientos de España emplean el DNle como sistema de autentificación o firma para el acceso a algún recurso, trámite, servicio o información.

Para ello se llevó a cabo un muestreo tomando tres ayuntamientos por provincia: el ayuntamiento con mayor población, el que tuviera una población más cercana a la media de los ayuntamientos de la provincia y el de menor población. Se tomó como referencia territorial la provincia por ser las diputaciones provinciales -o entidades equivalentes- las responsables de prestar los servicios de los municipios que no pudieran hacerlo. De hecho, el estudio incluyó las webs de las diputaciones provinciales para comprobar si así lo hacían.

Se establecieron las siguientes condiciones para considerar un "SÍ" en cada sitio web:

- al menos un recurso, trámite, servicio o información debe emplear el DNle como sistema de autentificación o firma del ciudadano;

- debe identificarse claramente que se acepta el DNle como sistema de autentificación o firma;

- no es necesario que el acceso al recurso, trámite, servicio o información esté alojado en el propio sitio web del ayuntamiento, pero sí que sea accesible desde la web oficial y que siga identificando, por el escudo o por el nombre, al ayuntamiento.
- en el caso de los sitios web de las diputaciones provinciales u otros organismos supramunicipales, los recursos, trámites, servicios o informaciones que acepten autentificación o firma con DNle deben ser explícitamente de ámbito municipal.

Un "NO" como resultado debe interpretarse como una de las siguientes posibilidades:

- no hay recursos, trámites, servicios o informaciones que requieran la autentificación o firma o, si los hay, no emplean o no explicitan la posibilidad de hacerlo con el DNle.

- no se ha localizado en el sitio web esta posibilidad, pero podría existir (la búsqueda ha sido exhaustiva, pero es imposible que esté exenta de errores).

\section{Segmentación de los sitios web}

- A partir de las estadísticas de población del INE de 2010 se identificaron tres municipios por provincia: 1) el de mayor población; 2) aquel cuya población fuera más cercana a la media de los municipios de la provincia; 3) el de menor población.

- Se identificaron todos los organismos supramunicipales con competencias para prestar servicios municipales delegados. Éstos son: diputaciones provinciales, cabildos insulares y comunidades autónomas uniprovinciales.

- En total se estudiaron las webs de 150 ayuntamientos y 2 ciudades autónomas. Esta muestra representa el 34\% de la población española.

- A lo anterior se añadieron los sitios web de 53 organismos supramunicipales.

El estudio se llevó a cabo durante del mes de febrero de 2011.

\section{Qué necesita el ciudadano}

Para hacer uso de la e-administración local el ciudadano, en principio, tan sólo necesita un ordenador con conexión a internet, un navegador web y un certificado electrónico reconocido por la web del ayuntamiento. Certificados electrónicos reconocidos por distintas administraciones hay varios, como el que expide la Fábrica Nacional de Moneda y Timbre (FNMT) o la Agència Catalana de Certificació. Pero los más extendidos en posesión de los ciudadanos, como veremos más adelante, son los que incorpora el DNle.

Para que un ciudadano pueda usar los certificados del DNIe en la web de un ayuntamiento necesita: un ordenador conectado a internet, un navegador web compatible (no lo son todos pero sí los más extendidos), un programa que ha de instalar una sola vez y que puede descargar desde la web del DNle, el PIN personal que garantiza el acceso a los certificados del DNle, el propio DNle y un lector de tarjetas inteligentes que cumpla el estándar ISO-7816.

\section{Qué necesita el ayuntamiento (o la diputación provincial)}

Si bien los requisitos para que un ciudadano pueda utilizar su DNle en la web de un ayuntamiento son pocos y relativamente sencillos, los que necesita un ayuntamiento para 


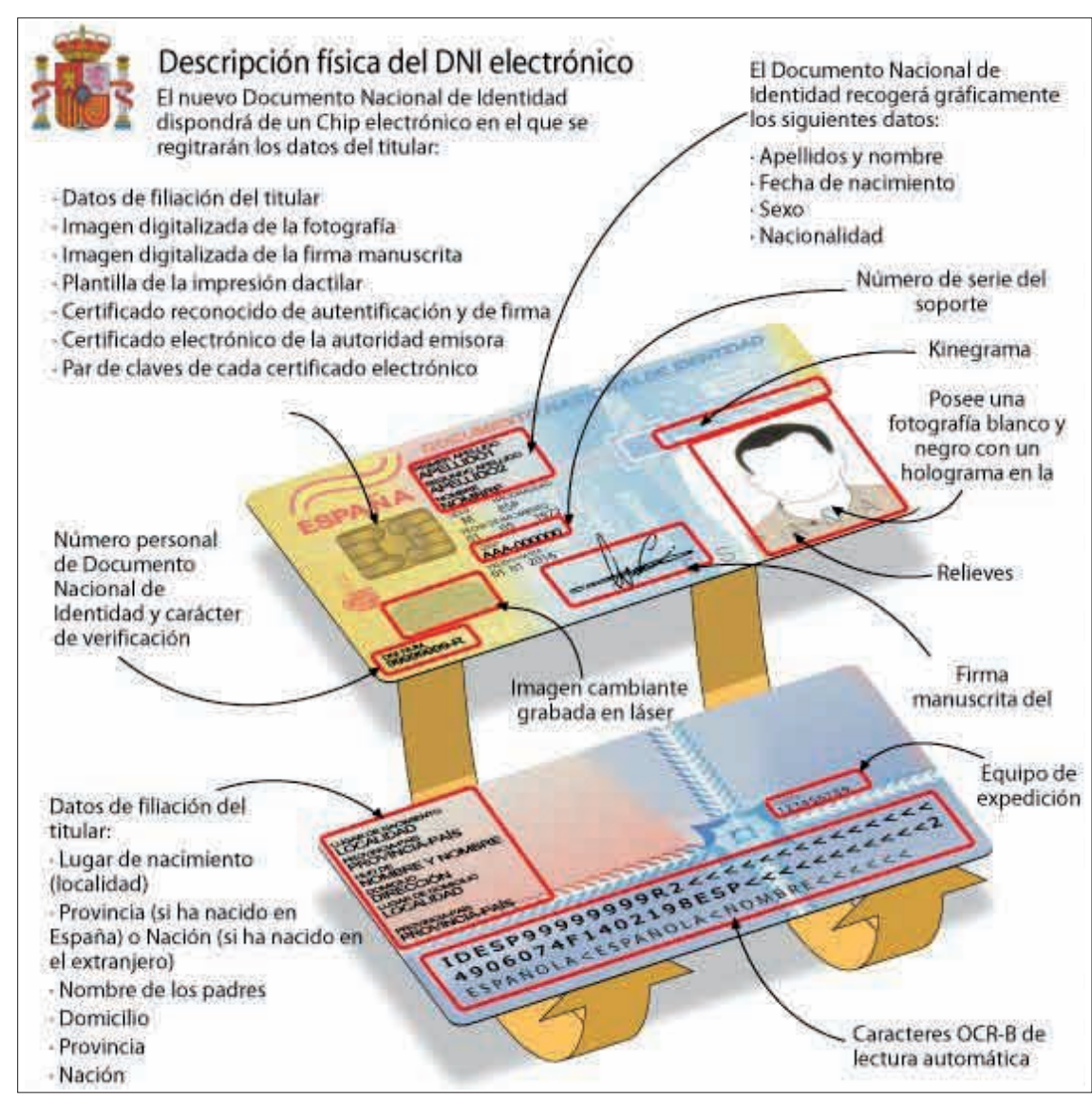

Gráfico 1. Descripción física del DNle. (Fuente: Ministerio del Interior, http://www.dnielectronico.es/)

diferentes de la autoridad de certificación. Mientras que quien certifica es el Ministerio del Interior a través de la Dirección General de la Policía, las autoridades de validación son la Fábrica Nacional de Moneda y Timbre (para ciudadanos, empresas y administraciones públicas) y el Ministerio de la Presidencia (exclusivamente para administraciones públicas).

La solución propuesta por el Ministerio de Política Territorial y Administración Pública (MPT) para la validación de un certificado electrónico desde la web de un ayuntamiento requiere, en primer lugar, que el ayuntamiento disponga de acceso a la red SARA (Sistema compuesto de aplicaciones y redes para las administraciones públicas), dependiente del propio MPT. Esta red, según la web del Ministerio, "permite la interconexión de las administraciones públicas, facilitando el intercambio de información y servicios entre ellas".

Uno de los servicios de la citada red es la Plataforma de validación y firma electrónica, asociada a la solución tecnológica@firma, desarrollada originalmente por la Junta de Andalucía y

implementar el uso del DNle en su web -si la tiene- son muchos y relativamente complejos. Entre otras cosas, necesitaría contar con un registro digital de entrada y salida de documentos. Con la intención de no hacer las cosas artificialmente complejas nos centraremos exclusivamente en los requisitos que se refieren al uso del DNle. Es decir, asumimos que el ayuntamiento tiene una web y la infraestructura necesaria para que se puedan realizar trámites o consultas que requieran algún tipo de autentificación o firma por parte del ciudadano (a lo que está, en cualquier caso, teóricamente obligado por ley).

Para que la web de un ayuntamiento pueda comprobar la validez de un certificado electrónico empleado por un ciudadano es necesario que pueda conectarse con la autoridad de validación del certificado. En el caso del DNle, con el fin de aislar la comprobación de la vigencia de un certificado electrónico de los datos de identidad de su titular, se ha optado por asignar funciones de validación a entidades posteriormente cedida al resto de las administraciones públicas. Actualmente el organismo responsable de @firma es la Dirección General para el Impulso de la Administración Electrónica, del MPT.

\section{El $27,5 \%$ de la población española entre 16 y 74 años dispone de DNle}

Dado que la implantación de lo antedicho puede ser compleja y costosa, la Ley 11/2007 de acceso electrónico de los ciudadanos a los servicios públicos prevé que, para que se hagan efectivos los derechos de los ciudadanos, "las diputaciones provinciales, o en su caso los cabildos y consejos insulares u otros organismos supramunicipales, podrán prestar los servicios precisos para garantizar tal efectividad en el ámbito de los municipios que no dispongan de los medios técnicos y organizativos necesarios para prestarlos".

\begin{tabular}{|c|c|c|}
\hline Elemento del ecosistema & Fuente (fecha) & $\%$ \\
\hline Población con acceso al menos a un servicio en web con su administración local usando DNle & Elaboración propia (febrero de 2011) & 83,0 \\
\hline Población con acceso al menos a un servicio en la web de su ayuntamiento usando DNle & Elaboración propia (febrero de 2011) & 74,0 \\
\hline Webs de diputaciones provinciales con al menos un servicio de ayuntamiento usando DNle & Elaboración propia (febrero de 2011) & 40,0 \\
\hline Web de ayuntamientos con al menos un servicio usando DNle & Elaboración propia (febrero de 2011) & 34,0 \\
\hline Población con DNle (entre 16 y 74 años) & INE (2010) & 27,5 \\
\hline Población con otros certificados electrónicos (entre 16 y 74 años) & INE (2010) & 9,0 \\
\hline Población con lector de DNle (entre 16 y 74 años) & INE (2009) & 2,0 \\
\hline
\end{tabular}

Tabla 1. El ecosistema de la e-administración local 


\section{Situación actual}

Expuesto el ecosistema necesario para que la e-administración local funcione con el DNle, para conocer la situación actual analizamos el estado de sus componentes clave: existencia de sistemas de autentificación o firma con DNle en los sitios web de los ayuntamientos, número de ciudadanos que disponen de este documento y número de ciudadanos que disponen de lector compatible con el mismo.

De los ayuntamientos de la muestra sólo un $34 \%$ presta en su web al menos un servicio que requiera el empleo del DNle. Pero esta cifra -pequeña- puede resultar engañosa porque, extrapolando los datos al conjunto de la población española, esos municipios representan el $74 \%$ de la población. De hecho, si se añaden los servicios a ayuntamientos prestados desde las webs de las diputaciones provinciales, aunque sólo lo hacen el $40 \%$ de las diputaciones, se cubre el $83 \%$ de la población española.

Como ya se ha dicho, existen varios certificados electrónicos que permiten tanto acreditar la identidad como firmar electrónicamente un documento con validez legal. Aunque no era el objetivo del estudio, durante la investigación de las webs de los ayuntamientos y diputaciones provinciales se detectó que varios de estos certificados electrónicos o bien convivían con el DNle o eran la única puerta de acceso al servicio prestado. Sin embargo, según datos del INE de 2010, los certificados electrónicos más difundidos son los propios del DNle. El $27,5 \%$ de la población española entre 16 y 74 años dispone de DNle frente al $9 \%$ que dispone de otros certificados electrónicos reconocidos.

Finalmente, para completar el conocimiento de la situación actual, los últimos de datos del INE respecto de la disponibilidad de lector compatible con el DNle son de 2009: el 2\% de la población española de entre 16 y 74 años dispone de tal lector. Curiosamente este dato estadístico no aparece en las encuestas de 2010.

\section{Conclusión}

Si la e-administración local debe hacerse realidad en algún momento, y una de sus piezas es el DNle, es necesario actuar en varios frentes.

El primero y más acusado es el de la disponibilidad de lectores de tarjetas inteligentes. Es el gran cuello de botella de todo el sistema. De nada sirve que los ciudadanos tengan en su bolsillo un DNle si no tienen el lector. Se podría actuar fa-

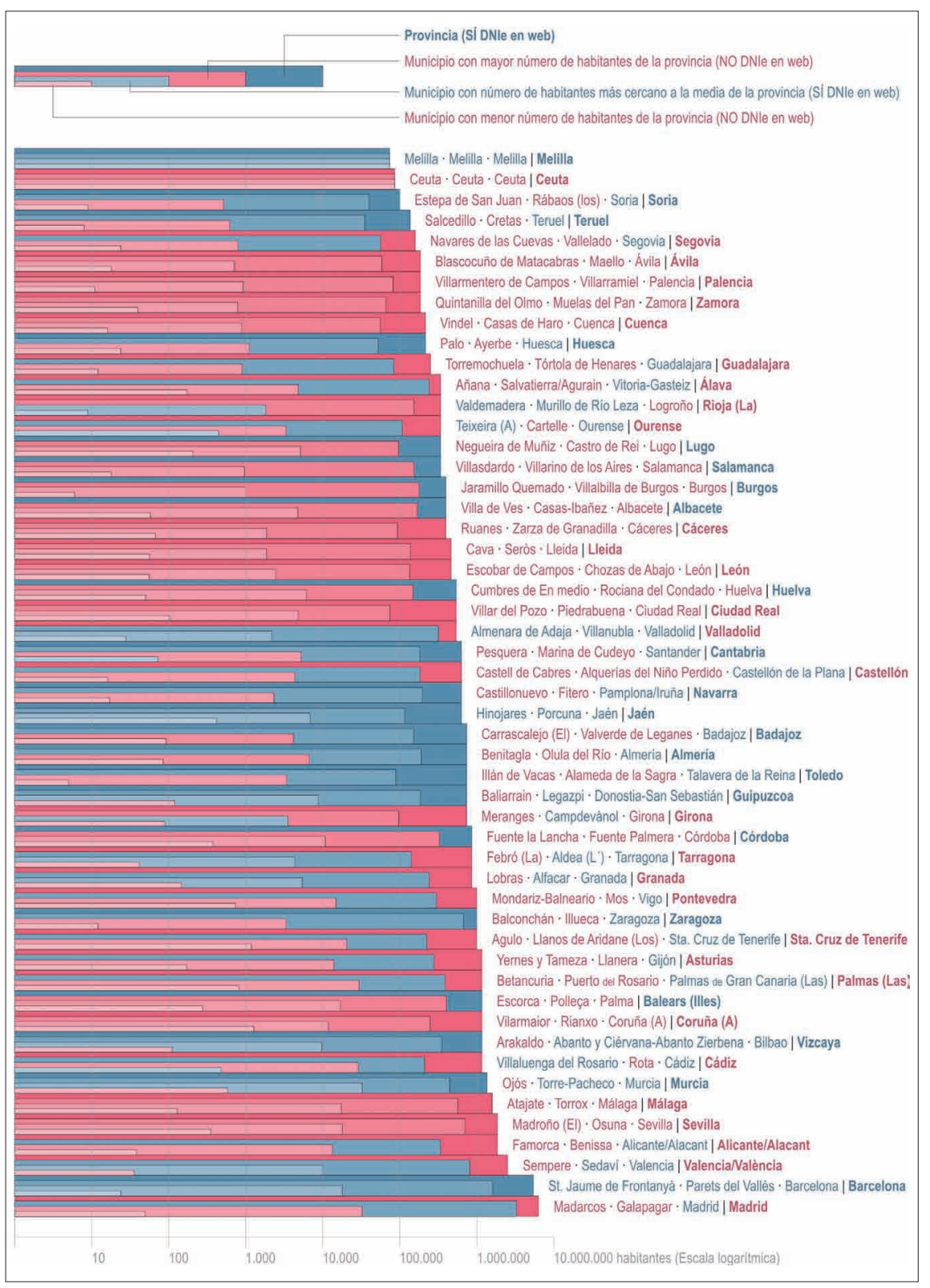

Gráfico 2. Ayuntamientos y diputaciones provinciales -o entidades equivalentes- que prestan en su web al menos un servicio que requiere el empleo del DNle

De nada sirve que los ciudadanos tengan en su bolsillo un DNIe si no tienen el lector

voreciendo de alguna forma la incorporación del dispositivo lector en los ordenadores que se venden en España.

El siguiente, por orden de importancia, es el de la disponibilidad del propio DNIe. De momento el porcentaje de población que dispone de él es bajo. Pero no parece que sea necesaria ninguna actuación especial: la propia naturaleza del ciclo de renovación del DNI actuará en este sentido.

Por último, es necesario actuar en la propia administración local. Aunque el porcentaje de población que ya podría emplear el DNle en los sitios web de las administraciones locales es alto, el porcentaje de ayuntamientos que ofrecen esta posibilidad es bajo.

$\mathrm{O}$, tal vez, haya que renunciar al DNle como sistema de autentificación y firma electrónica. El certificado electrónico 
emitido por la Fábrica Nacional de Moneda y Timbre tiene la misma validez para autentificación y firma que el DNle y se puede llevar en una memoria USB. Está reconocido en la solución tecnológica @firma del MPT y el dispositivo lector, un puerto USB, sí que está presente en la mayor parte de los ordenadores.

\section{Bibliografía}

Baiget, Tomàs. "DNI electrónico (DNle)". Anuario ThinkEPI, 2007, pp. 203-204.

INE. Encuesta sobre equipamiento y uso de tecnologías de la información y comunicación en los hogares 2010.

http://www.ine.es/jaxi/menu.do?type=pcaxis\&path=/t25/ p450/a2010/\&file $=$ pcaxis

INE. Encuesta sobre equipamiento y uso de tecnologías de la información y comunicación en los hogares 2009.

http://www.ine.es/jaxi/menu.do?type=pcaxis\&path=/t25/ p450/a2009\&file $=$ pcaxis

Ley 11/2007, de 22 de junio, de acceso electrónico de los ciu- dadanos a los servicios públicos. Boletín Oficial del Estado, n. 150, 23 de junio de 2007, pp. 27150-27166.

Ministerio de Administraciones Públicas. "Red SARA: La red de las administraciones públicas españolas". En: Jornadas de formación sobre implantación de la Ley 11/2007 en los entes locales. Palencia, 2008.

http://www.jcyl.es/web/jcyl/pr/ds/MunicipiosDigitales/ pdf;charset=UTF-8/144/334/INTEROPERABILIDAD.pdf/_ ?asm=jcyl

Plataforma de validación de firma electrónica @firma http://administracionelectronica.gob.es/?_nfpb=true\&_ pageLabel=PAE_PG_CTT_General\&langPae=es\&iniciativa $=$ 190

Portal oficial sobre el DNI electrónico http://www.dnielectronico.es/

Red SARA

http://www.csi.map.es/csi/pg5r12.htm

@firma. Plataforma de validación y firma electrónica http://www.csi.map.es/csi/pg5a12.htm

\section{ProQuest Historical Newspapers ${ }^{\mathrm{TM}}$}

\section{El Archivo Digital definitivo}

Acceso a las portadas, artículos, editoriales,

fotografías, anuncios, anuncios de boda y

de compromiso, dibujos animados,

anuncios de nacimiento y defunciones,

reseñas de libros y películas, cartas y

mucho más, que nos permiten ser testigos tanto de la vida de la gente común, como de los grandes eventos. ProQuest

Historical Newspapers, es el archivo digital definitivo, ofrece más de tres siglos y más de 26 millones de páginas digitalizadas de EE.UU. y de noticias internacionales,

información de primera mano y opiniones que dan vida a la historia.

Para solicitar un acceso gratuíto, vaya a www.proquest.com/go/hnp o envie un email a newspapers@proquest.co.uk incluyendo la referencia AD 54311

Para más información, vaya a www.proquest.com

\section{¡Nuevo en 2011 !}

ProQuest Historical

Newspapers puede ser buscado simultáneamente con ProQuest Newsstand a través de la nueva plataforma de ProQuest, lo que permite acceder tanto a la

información histórica como a la actual.

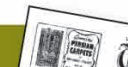

